\title{
EFECTOS DE LA DENSIDAD DE SIEMBRA Y EL DESDOBLE SOBRE EL CRECIMIENTO Y LA SUPERVIVENCIA EN UN CULTIVO SUSPENDIDO EXPERIMENTAL DEL MEJILLÓN Mytilus platensis D’ORBIGNY, 1842, EN EL GOLFO SAN JORGE, ARGENTINA
}

\author{
Bernardo G. Marques ${ }^{1,3,4}$, Tomás E. Isola ${ }^{1,3}$, Gabriel Soto ${ }^{2,4}$ y Héctor E. Zaixso ${ }^{1, \dagger}$ \\ ${ }^{1}$ Instituto de Desarrollo Costero (IDC), Universidad Nacional de la Patagonia San Juan Bosco \\ (UNPSJB), Ruta Provincial No 1 km 4 s/n, Comodoro Rivadavia, Argentina \\ correo electrónico: bernardo.gabriel.marques@gmail.com \\ ${ }^{2}$ Departamento de Química, Facultad de Ciencias Naturales, Universidad Nacional de la Patagonia \\ San Juan Bosco (UNPSJB), Ruta Provincial N $1 \mathrm{~km} 4 \mathrm{~s} / \mathrm{n}$, Comodoro Rivadavia, Argentina \\ ${ }^{3}$ CIT-Golfo San Jorge, CONICET, Ruta Provincial No $1 \mathrm{~km} 4 \mathrm{~s} / \mathrm{n}$, Comodoro Rivadavia, Argentina \\ ${ }^{4}$ Departamento de Matemática, Facultad de Ingeniería, Universidad Nacional de la Patagonia San \\ Juan Bosco (UNPSJB), Ruta Provincial No 1 km 4 s/n, Comodoro Rivadavia, Argentina
}

\begin{abstract}
RESUMEN. En los últimos años, ha habido un renovado interés en el desarrollo del cultivo de mejillones en la Argentina. Sin embargo, esta actividad todavía tiene lugar a pequeña escala o de forma experimental en la región patagónica. Conocer los efectos de las densidades iniciales y el desdoble sobre el crecimiento y la supervivencia en un cultivo experimental es información de base necesaria para el desarrollo de experiencias que permitan estudiar parámetros productivos en cultivos a mayor escala. Por estos motivos, este trabajo tuvo como objetivo analizar el efecto de las densidades iniciales y el desdoble sobre el crecimiento y la supervivencia del mejillón Mytilus platensis en un cultivo suspendido experimental en la región costera central del Golfo San Jorge, Argentina. Para ello, se estudiaron dos técnicas de cultivo, con y sin desdoble, durante un período de 9 meses, a dos densidades iniciales (400 y 700 ind. $\mathrm{m}^{-1}$ ). La semilla fue obtenida de un criadero artificial. Se monitorearon talla, peso y supervivencia de los mejillones. El mayor crecimiento en talla y peso, y la supervivencia más elevada se observaron en la estrategia sin desdoble con la densidad más baja. En esta estrategia, los animales alcanzaron una talla promedio mayor a $65 \mathrm{~mm}$, llegando a la talla comercial en menos de un año. Estos resultados permiten concluir que, a pequeña escala, la estrategia sin desdoble es la recomendada para cultivar mejillones en la región a una densidad de 400 ind. $\mathrm{m}^{-1}$ durante un mínimo de 9 meses.
\end{abstract}

Palabras clave: Cultivo de mejillón, desdoble, crecimiento, supervivencia.

\section{EFFECTS OF STOCKING DENSITY AND THINNING-OUT ON GROWTH AND SURVIVAL IN AN EXPERIMENTAL SUSPENDED CULTURE OF THE MUSSEL Mytilus platensis D'ORBIGNY, 1842, IN SAN JORGE GULF, ARGENTINA}

\begin{abstract}
There has been a renewed interest in the development of mussel farming in Argentina in the recent years. However, this activity still takes place on a small scale or experimentally in the Patagonian region. Knowing the effects of initial densities and thinning-out on growth and survival at an experimental scale is a necessary basic infor-
\end{abstract}

†El Dr. Héctor E. Zaixso falleció el 28 de abril de 2015. 
mation for the development of experiences that allow the study of productive parameters in larger-scale culture. For these reasons, this work aimed to analyze the effect of initial densities and thinning-out on the growth and survival of the mussel Mytilus platensis in an experimental suspended culture in the central coastal region of San Jorge Gulf, Argentina. Two cultivation techniques were studied, with and without thinning-out, for 9 months at two initial densities (400 and 700 ind. $\mathrm{m}^{-1}$ ). Seed was supplied by a hatchery. Size, weight and survival of the mussels were monitored. The best performance was achieved without thinning-out at the lower density. Additionally, animals reached an average of more than $65 \mathrm{~mm}$ with this strategy, obtaining commercial sizes in less than one year. These results allow us to conclude that the thinning-out strategy is recommended for cultivating mussels on a small scale in this region at a density of 400 ind. $\mathrm{m}^{-1}$ for a minimum of 9 months.

Key words: Mussel culture, thinning-out, growth, survival.

\section{INTRODUCCIÓN}

Durante las últimas décadas, el cultivo suspendido de mejillón ha sido una actividad comercial de gran expansión en países con litorales marítimos apropiados (Ross et al. 2013). En la Argentina, esta actividad aún se realiza de manera esporádica, a pequeña escala o de forma experimental (RMCP 2013; Isola 2017). En 2014, las importaciones realizadas por la Argentina de productos derivados del cultivo de mejillón superaron las $1.000 \mathrm{t}$, con un valor de más de US\$ 2.500.000, siendo Chile el país que acapara casi la totalidad $(99,7 \%)$ de dichas importaciones (PROCHILE 2014). Por otro lado, el cultivo suspendido de diversos moluscos bivalvos en la Argentina (entre los que se encuentra el mejillón Mytilus platensis d'Orbigny, 1842 y la cholga Aulacomya atra (Molina, 1782)), alcanzó un producción de $16 \mathrm{t}$ en 2015 (Panné Huidobro 2015). Esta enorme brecha entre los niveles de demanda interna y la producción nacional revela un importante nicho para el desarrollo de actividades productivas relacionadas al cultivo del mejillón.

Estudios en todo el mundo han demostrado la importancia de la densidad de siembra inicial de animales (individuos por metro, ind. $\mathrm{m}^{-1}$ ) en el desarrollo de un cultivo suspendido de mejillón (Alunno-Bruscia et al. 2000; Cubillo et al. 2012a; Pérez-Camacho et al. 2013; Bordon et al. 2014). Altas densidades iniciales generan competencia por espacio y/o alimentos (Fréchette y Lefaivre
1990), así como mortalidad asociada a la competencia intraespecífica a medida que los animales se desarrollan (Fréchette et al. 1996; Fréchette y Bacher 1998; Alunno-Bruscia et al. 2000; Guiñez 2005; Fréchette et al. 2011; Fuentes-Santos et al. 2014). Se han registrado también efectos densodependientes sobre el desarrollo individual y el aumento en las pérdidas por desprendimiento (Lauzon-Guay et al. 2005a; Cubillo et al. 2012a, $2012 b$ ). Por estas razones, conocer cuáles estrategias de manejo y densidades iniciales son las adecuadas para reducir la competencia intraespecífica, optimizando el crecimiento y la supervivencia en un cultivo experimental, es información de base necesaria para el adecuado desarrollo de futuras experiencias a escala comercial que permitan analizar niveles productivos en la región costera central del Golfo San Jorge.

Por lo general, dos estrategias de manejo son utilizadas en el cultivo suspendido de mejillón para mitigar los efectos contraproducentes de la densidad. Una de esas estrategias, conocida como cultivo con desdoble (thinning-out, Pérez-Camacho et al. 2013), consiste básicamente en engordar animales jóvenes a altas densidades en las estructuras de cultivo durante un determinado periodo de tiempo (fase de cría o pre-engorde). Pasado este tiempo, los animales son retirados de las estructuras de cultivos y re-colocados en las mismas a densidades menores hasta el tiempo de cosecha (fase de engorde). Este proceso se lleva a cabo para controlar la densidad de individuos en las unidades de cultivo y así favorecer el crecimiento y reducir las pérdidas por desprendimiento. 
Esta estrategia posee variaciones de acuerdo al lugar donde se lleve a cabo esta práctica. En España, por ejemplo, en el cultivo del mejillón $M$. galloprovincialis Lamarck, 1819, el proceso de desdoble consta de tres etapas:

1) Obtención de la semilla: consiste en obtener (por captación en cuerdas o redes colectoras y/o recolección del ambiente natural) juveniles con tallas que oscilan entre 15 y $30 \mathrm{~mm}$;

2) Fase de pre-engorde: estos animales se colocan en las cuerdas de cultivo a altas densidades por metro de cuerda durante un período que oscila entre los 3 y 6 meses;

3) Fase de engorde: luego de este período, esas cuerdas se retiran del agua, los mejillones son separados de las cuerdas y re-encordados a densidades menores (desdoble), haciéndolos crecer en estas cuerdas hasta el momento de cosecha (Pérez-Camacho et al. 2013).

En el cultivo del chorito (M. chilensis, Hupé, 1854) en Chile, se utiliza un proceso similar al español, en el cual las dos primeras etapas están comprendidas en una, ya que la semilla se obtiene por captación natural en cuerdas colectoras, y luego que los individuos alcanzan un tamaño entre 10 y $30 \mathrm{~mm}$ en esas cuerdas, se pone en práctica la fase de engorde (Uriarte 2008).

Otra técnica o estrategia de cultivo consiste en colocar individuos en densidades específicas en las cuerdas y dejarlos hasta el momento de cosecha. Este proceso se conoce como cultivo sin desdoble (Pérez-Camacho et al. 2013). Este tipo de producción minimiza las horas hombre de trabajo y generalmente se usa en países tropicales (Gosling 1992). Estudios comparando la efectividad entre cultivos con y sin desdoble sugieren que este último reduce el tiempo de cultivo y el trabajo asociado con este proceso, con el consiguiente aumento de la productividad (PérezCamacho et al. 2013).

En la Argentina, el cultivo de mejillón se ha realizado a pequeña escala en emprendimientos que no han contemplado, de forma sistemática, el efecto de la densidad de siembra sobre la productividad general del cultivo (RMCP 2013). En la región costera norte y central de la Patagonia argentina, la técnica de cultivo suspendido que ha brindado los mejores resultados es el cultivo en longline subsuperficiales (Bertolotti et al. 1987; Zaixso y Bala 1993; Pascual y Zampatti 1998; Elvira et al. 2000; Zaixso y Lizarralde 2001; Isola y Zaixso 2012; RMCP 2013). Zaixso y Lizarralde (2001) realizaron estudios experimentales sobre el crecimiento, supervivencia y biomasa cosechable a tres densidades de engorde diferentes en un cultivo con desdoble en el Golfo Nuevo $\left(42^{\circ} 46^{\prime} \mathrm{S}, 65^{\circ} 02^{\prime} \mathrm{W}\right)$. Este trabajo mostró que en un cultivo con desdoble el aumento de la densidad inicial afecta negativamente el crecimiento y la supervivencia.

En la región central del Golfo San Jorge, se estudió el efecto de la densidad inicial sobre el crecimiento y la supervivencia en un cultivo sin desdoble con una variante del método de encordado francés (González et al. 1974), a tres densidades iniciales diferentes (Isola y Zaixso 2012). Los resultados mostraron que el aumento de la densidad inicial afecta negativamente el crecimiento y la supervivencia. Sin embargo, en la región no existe información que unifique, en un único estudio, los efectos conjuntos del desdoble y la densidad de siembra sobre el crecimiento y la supervivencia del mejillón y compare estos resultados frente a un cultivo sin la práctica del desdoble a las mismas densidades iniciales.

Desarrollar un cultivo con y sin la práctica del desdoble, a diferentes densidades iniciales de engorde y estudiar sus efectos sobre el crecimiento y la supervivencia del mejillón, es parte de la información de base necesaria para definir estrategias de manejo en futuros emprendimientos a mayor escala que optimicen el uso del recurso (semilla) y los niveles de producción. Los resultados de un estudio de estas características aportan información para el desarrollo de políticas públicas relacionadas tanto con la sustentabilidad 
como con la expansión futura de esta actividad en la región, en concordancia con las directivas actuales sobre producción acuícola en el país (InfoLEG 2017).

Por estas razones, el presente estudio tuvo como objetivo comparar el crecimiento y la supervivencia del mejillón $M$. platensis, en cultivo experimental con y sin la práctica del desdoble, a diferentes densidades iniciales en la región costera central del Golfo San Jorge.

\section{MATERIALES Y MÉTODOS}

El estudio se realizó en el área costera central del Golfo San Jorge, Chubut, Argentina. Este golfo se caracteriza por ser semiabierto con un área que abarca aproximadamente $39.340 \mathrm{~km}^{2}$. Los valores de salinidad registrados se ubican dentro del rango comprendido entre los 33 a 33,6 (Akselman 1996; Fernández et al. 2005, 2008). La productividad primaria del área registra dos picos, uno a comienzos de la primavera y otro a comienzos del otoño (Carreto et al. 2007). La experiencia se realizó en Playa Belvedere ( $45^{\circ} 59^{\prime} 12^{\prime \prime} \mathrm{S}, 67^{\circ} 33^{\prime} 17^{\prime \prime} \mathrm{W}$, Figura 1), a una profundidad promedio de $15 \mathrm{~m}$ en relación a la altura media de las mareas. Para la realización de los ensayos se instaló un longline experimental sub-superficial de $100 \mathrm{~m}$ de longitud efectiva, fondeado en sus extremos con estructuras de hormigón de base circular de 1,5 t cada una. Mensualmente y durante el período de estudio (mayo de 2016 a febrero de 2017), se registró la concentración de clorofila $a(\mathrm{Chl} a)$, materia orgánica particulada (MOP), temperatura del agua y salinidad. La estimación de Chl $a$ se realizó a través de imágenes del satélite Aqua Modis $4 \mathrm{~km}$, y la temperatura del agua de mar y salinidad se registraron con una sonda multiparamétrica YSI 556 MPS. Para determinar el contenido de MOP se tomaron 3 muestras mensuales de 11 de agua de mar que fueron filtradas uti- lizando filtros de microfibra de vidrio Munktell MG-160 de $47 \mathrm{~mm}$. El contenido de MOP se determinó luego de calcinar en una mufla los filtros a una temperatura de $550{ }^{\circ} \mathrm{C}$ siguiendo el protocolo descripto por Strickland y Parsons (1965).

\section{Experiencia de cultivo}

Los mejillones utilizados en el experimento fueron juveniles de $M$. platensis provenientes de un criadero ubicado en la localidad de Bahía Camarones, Provincia del Chubut, Argentina. La semilla presentó una talla promedio de $27.37 \mathrm{~mm}$ $\left(\mathrm{s}^{2}=25,14, \mathrm{n}=1.100\right)$ y un peso seco promedio de $0,069 \mathrm{~g}\left(\mathrm{~s}^{2}=0,00215\right.$, sub-muestra al azar de 100 individuos). En mayo de 2016 se confeccionaron los encordados utilizando un sistema mixto francés-español, el cual consiste en encordar por el método francés (Gonzales et al. 1974) colocando palillos de madera para distribuir la carga de la cuerda y que funcionan como soportes, como en el método español (Gonzales et al. 1974).

Para estudiar los efectos de la técnica de manejo y las densidades iniciales sobre el crecimiento y supervivencia en un cultivo experimental de mejillón, se analizaron dos técnicas de cultivo: con y sin desdoble a dos densidades iniciales de engorde. Para la experiencia sin desdoble (ESD), se confeccionaron ocho cuerdas de $1 \mathrm{~m}$ de longitud con una densidad inicial de 400 ind. $\mathrm{m}^{-1}$ cada una (ESD-400) y ocho cuerdas de $1 \mathrm{~m}$ con una densidad inicial de 700 ind. $\mathrm{m}^{-1}$ (ESD-700). Para la experiencia con desdoble (ECD), se prepararon nueve cuerdas de $1 \mathrm{~m}$ de longitud con una densidad inicial de 1.600 ind. $\mathrm{m}^{-1}$ (ECD-1.600, cuerdas de cría). La elección de estas densidades iniciales de engorde se basó en los resultados de un trabajo previo en la región (Zaixso y Lizarralde 2001), el cuál mostró que el engorde a altas densidades iniciales (en un cultivo exclusivamente bajo las técnica con desdoble) causa elevados desprendimientos de individuos, amenazando la rentabilidad del cultivo. 


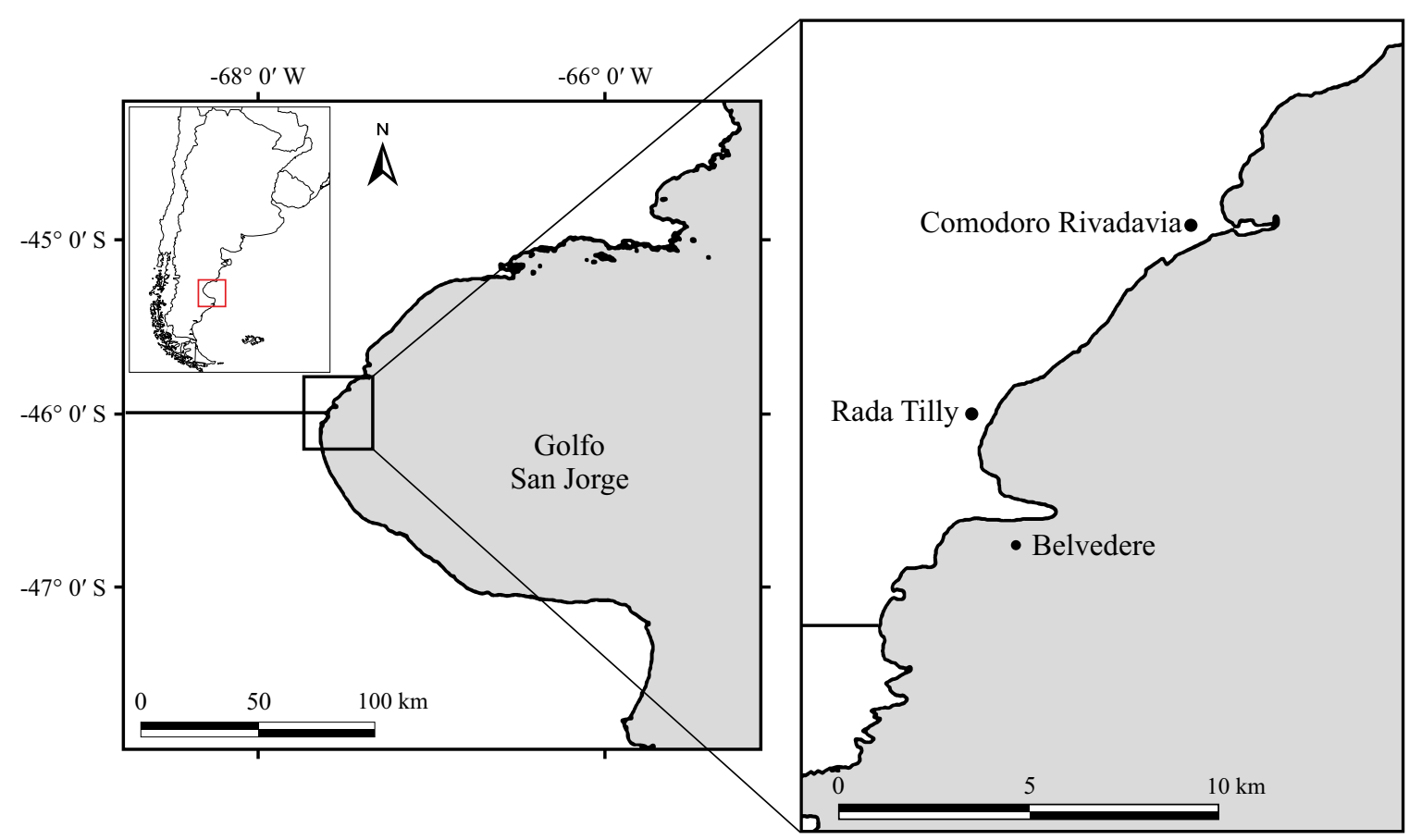

Figura 1. Ubicación de la zona de estudio. Playa Belvedere. Figure 1. Location of the study area. Belvedere beach.

Para evitar posibles interacciones entre las cuerdas, estas fueron colocadas en el longline con una separación de $3 \mathrm{~m}$ entre ellas y de forma alternada siguiendo el siguiente esquema: ESD400, ESD-700, ECD-1.600. El experimento comenzó en mayo de 2016 y finalizó en febrero de 2017 (nueve meses). Se muestrearon cuerdas de cultivo cada tres meses tomando al azar dos cuerdas correspondientes a cada densidad inicial (en ambas estrategias) para medir parámetros de crecimiento y supervivencia.

En la ECD-1.600, tres meses después de iniciado el experimento (agosto), se realizó el desdoble y se extrajeron las nueve cuerdas correspondientes a esta serie experimental. De estas cuerdas, dos se usaron para medir parámetros de crecimiento y supervivencia, mientras que con las restantes (siete) se armaron doce cuerdas de engorde: seis con una densidad inicial de 400 ind. $\mathrm{m}^{-1}$ (ECD-400) y otras seis con una densidad inicial de 700 ind. $\mathrm{m}^{-1}$ (ECD-700). Los individuos rema- nentes, una vez finalizado el armado de las cuerdas de engorde y la toma de muestras, fueron reencordados y colocados nuevamente en la línea de cultivo para su utilización con otros fines no vinculados al presente estudio. Luego del desdoble, las cuerdas se distribuyeron en el longline siguiendo el siguiente esquema: ESD-400, ECD400, ESD-700, ECD-700. En cada fecha de muestreo y en cada cuerda retirada, se realizó un conteo de todos los individuos vivos y se tomaron muestras aleatorias de 50 mejillones. A cada individuo se le midió la talla (distancia umbo-borde posterior), se le extrajo el tejido blando y se secó en estufa a $80{ }^{\circ} \mathrm{C}$ hasta alcanzar peso constante, para luego pesarlo en balanza analítica.

\section{Análisis de supervivencia}

La supervivencia por cuerda se definió como el número de individuos vivos que permanecieron en la cuerda al momento del muestreo. La diferencia entre el número inicial y la supervivencia 
(sumado a los pocos individuos muertos hallados en cada cuerda) se consideró como individuos desprendidos.

En cada muestreo la supervivencia se estimó como el promedio de las dos cuerdas correspondientes a cada serie experimental (ESD-400, ESD-700 y ECD-1.600 a los 3 meses; y ESD400, ESD-700, ECD-400 y ECD-700 en los restantes muestreos). Con estos datos se calculó la supervivencia porcentual con respecto a las densidades iniciales correspondientes. A los tres meses y dentro de cada estrategia (en cada fecha de muestreo), se analizó la relación entre densidad inicial y porcentaje de supervivencia a través de una prueba de independencia $\chi^{2}(\alpha=0,05$, Sokal y Rohlf 1995).

Para analizar la independencia entre las densidades iniciales y el porcentaje de supervivencia al momento de cosecha (nueve meses) entre todas las series experimentales (ESD y ECD), se estimó la probabilidad de supervivencia total correspondiente a cada serie experimental. Para esto, es necesario cuantificar la supervivencia total en la ECD cuando se consideran en conjunto la fase de pre-engorde y la fase de engorde. Por esta razón, se propuso un método teórico para calcular la probabilidad de supervivencia total en ambas experiencias. Desde este punto de vista, la probabilidad de supervivencia total se puede expresar como el producto de dos probabilidades: la probabilidad de sobrevivir a la fase de pre-engorde a una determinada densidad inicial y la probabilidad de sobrevivir a la fase de engorde a una determinada densidad inicial, dado que sobrevivió a la fase de pre-engorde. Por lo tanto, la probabilidad de sobrevivir a todo el proceso se define como:

$$
P(S T)=P(F \mid B) \cdot P(B)
$$

donde $P(S T)$ es la probabilidad de supervivencia total (la probabilidad de supervivencia para todo el proceso); $P(B)$ es la probabilidad de sobrevivir a la fase de pre-engorde a una densidad inicial B; y $P(F \mid B)$ es la probabilidad de sobrevivir a la fase de engorde a una densidad inicial de engorde $F$ condicionada a la fase de pre-engorde a una densidad inicial B.

En el caso de la ESD, $P(B)=1$ ya que no se realizó desdoble y $P(F \mid B)$ es, en este caso, sólo la probabilidad de sobrevivir a la fase de engorde a una densidad inicial de engorde $F(P(F)$ ). Las probabilidades $P(F \mid B), P(B)$ y $P(F)$ se estimaron a partir de los valores porcentuales de supervivencia observados. Con esta información se estimó el porcentaje de supervivencia total. La dependencia entre cada tipo de tratamiento y el porcentaje de supervivencia total obtenido se analizó a través de una prueba de independencia $\chi 2(\alpha=0,05)$.

\section{Análisis de crecimiento}

En cada fecha de muestreo, el tamaño promedio (talla y peso) asociado a cada densidad inicial se estimó como el promedio de las muestras correspondientes.

En el caso de la talla, y para las tres densidades determinadas al comienzo del experimento (ECD-1.600, ESD-400 y ESD-700), se analizaron si existían diferencias significativas en el crecimiento después de tres meses a través de un ANOVA de una vía con un test de Tukey a posteriori $(\alpha=0,05)$. Se testearon previamente los supuestos de normalidad y homocedasticidad a partir de una prueba de Shapiro-Wilks $(\alpha=0,05)$ y Bartlett $(\alpha=0,05)$, respectivamente. En las fechas de muestreo restantes se analizaron diferencias significativas en la talla dentro de cada estrategia (ESD y ECD) a través de una prueba $t$ de Welsch (Sokal y Rohlf 1995), ya que todas las distribuciones de tallas resultaron normales Shapiro-Wilks $(\alpha=0.05)$ pero las varianzas presentaron heterogeneidad (Bartlett, $\alpha=0,05$ ).

Para comparar la talla a los nueve meses entre las cuatro series experimentales, dado que las varianzas resultaron heterogéneas (prueba de Bartlett, $\alpha=0,05)$, se utilizó una prueba de Kruskal-Wallis para analizar si existían diferencias significativas, y una prueba a posteriori de Dunn 
con el ajuste por el método FWER (Family-Wide Error Rate $)$ de Holm $(\alpha=0,05)$ para analizar qué series experimentales diferían entre sí.

La existencia de diferencias significativas para el crecimiento en peso seco a los tres meses, en las tres densidades determinadas al comienzo del experimento, se analizó mediante una prueba de Kruskal-Wallis debido a que los datos resultaron no normales (Shapiro-Wilks, $\alpha=0,05$ ) y heterocedásticos (Levene, $\alpha=0,05$ ). Para determinar qué condición inicial presentaba el mejor rendimiento, se realizó una prueba a posteriori de Dunn con el ajuste por el método FWER (FamilyWide Error Rate) de Holm $(\alpha=0,05)$.

En las fechas de muestreo restantes, dentro de cada estrategia, las diferencias significativas en los pesos secos se estudiaron mediante una prueba de Mann-Whitney para muestras no pareadas (Mann y Whitney 1947, $\alpha=0,05$ ), ya que no se cumplieron las hipótesis de normalidad (Shapiro-Wilks, $\alpha=0,05$ ) ni homogeneidad de varianzas (Levene, $\alpha=0,05)$. Para comparar los pesos finales de las cuatro series experimentales entre sí, dado que las muestras presentaron distribuciones no normales (Shapiro-Wilks, $\alpha=0,05)$ y varianzas heterogéneas (Levene, $\alpha=0,05$ ), se utilizó una prueba de Kruskal-Wallis para detectar diferencias significa- tivas entre las distribuciones y un test a posteriori de Dunn, con el ajuste por el método FWER de Holm $(\alpha=0,05)$, para analizar qué serie experimental presentaba el mejor resultado.

\section{RESULTADOS}

\section{Variables ambientales}

Las concentraciones de MOP registraron su menor valor $\left(4,3 \mathrm{~g} \mathrm{~m}^{-3}\right)$ en julio de 2016 y los mayores valores se observaron hacia finales de la primavera, con un máximo en noviembre de $2016\left(9,3 \mathrm{~g} \mathrm{~m}^{-3}\right)$. Para la Chl $a$, la menor concentración $\left(1,55 \mathrm{mg} \mathrm{m}^{-3}\right)$ se observó en agosto de 2016. Durante los meses de primavera verano se observó un aumento en las concentraciones de Chl $a$ con un máximo en noviembre de 2016 $\left(9,23 \mathrm{mg} \mathrm{m}^{-3}\right)$.

La salinidad registró un valor máximo de 34,75 ppm en octubre de 2016 y un mínimo de 33,92 ppm en noviembre del mismo año. La temperatura registró su valor mínimo en agosto de 2016 con $7,48{ }^{\circ} \mathrm{C}$ y su máximo en febrero de 2017 con $16,77^{\circ} \mathrm{C}$ (Tabla 1 ). La falta de datos en alguno de

Tabla 1. Variables ambientales recolectadas a lo largo de la experiencia.

Table 1. Environmental variables measured throughout the experience.

\begin{tabular}{lcccc}
\hline Mes & MOP $\left(\mathrm{g} \mathrm{m}^{-3}\right)$ & $\mathrm{Chl} a\left(\mathrm{mg} \mathrm{m}^{-3}\right)$ & Salinidad $(\mathrm{ppm})$ & Temperatura $\left({ }^{\circ} \mathrm{C}\right)$ \\
\hline Mayo & 5,2 & 8,74 & 34,26 & 10,89 \\
Junio & 7,9 & - & 34,22 & 9,42 \\
Julio & 4,3 & 3,19 & 34,16 & 7,8 \\
Agosto & 5,15 & 1,55 & - & 7,48 \\
Septiembre & 7,46 & 1,93 & 34,15 & 9,43 \\
Octubre & 5,1 & 8,67 & 34,75 & 9,97 \\
Noviembre & 9,3 & 9,23 & 33,92 & 13,9 \\
Diciembre & 8,3 & 5,15 & 34,14 & 12,88 \\
Enero & 5,5 & 7,27 & - & 13,08 \\
Febrero & 4,4 & 4,68 & 34,07 & 16,77 \\
\hline
\end{tabular}


los meses para alguna de las variables se debió a un error de calibración de la sonda o la falta de datos satelitales.

\section{Experiencia de cultivo}

\section{Análisis de supervivencia}

Del análisis de los datos de supervivencia mostró que el porcentaje de supervivencia después de tres meses fue independiente de las densidades iniciales (prueba de independencia $\chi 2, p>0,05$, $\mathrm{GL}=2$ ) (Tabla 2).

En los restantes muestreos, dentro de la ESD se observó independencia entre las densidades ini- ciales y el porcentaje de supervivencia durante los dos primeros muestreos (3 y 6 meses, prueba de independencia $\chi 2, p>0,05, \mathrm{GL}=1)$. La supervivencia final mostró dependencia con respecto a las densidades iniciales (prueba de independencia $\chi 2, \mathrm{p}<0,0001, \mathrm{GL}=1)$, presentando el mayor valor en la ESD-400 (77\%). En los muestreos correspondientes a la ECD, se observó dependencia entre la densidad inicial y el porcentaje de supervivencia a lo largo de toda la experiencia (prueba de independencia $\chi 2, \mathrm{p}<0,05, \mathrm{GL}=1$ ), presentando la ECD-700 los mayores valores porcentuales de supervivencia. Estos porcentajes corresponden solo a la fase de engorde.

Tabla 2. Promedio y porcentaje de supervivencia en cada uno de los experimentos. Los porcentajes de supervivencia total considerando las fases de pre-engorde y engorde se muestran en negrita. Para cada fecha de muestreo, independencia entre densidad inicial y supervivencia dentro de cada estrategia se denota con la misma letra. Independencia entre estrategias y supervivencia se denota con * (prueba de independencia $\chi 2, \alpha=0,05)$. (\#): porcentaje de supervivencia de la fase de engorde solamente.

Table 2. Average and percentage of survival in each of the experiments. Percentages of total survival considering the pre-fattening and fattening phases are shown in bold. For each sampling date, independence between initial density and survival within each strategy is denoted with the same letter. Independence between strategies and survival is denoted with * (test of independence $\chi 2, \alpha=0.05)$. (\#): percentage of survival of the fattening phase only.

\begin{tabular}{|c|c|c|c|c|}
\hline \multirow[b]{2}{*}{3 meses } & \multicolumn{2}{|c|}{ Experiencia sin desdoble } & \multicolumn{2}{|c|}{ Experiencia con desdoble } \\
\hline & 400 ind. $\mathrm{m}^{-1}$ & 700 ind. $\mathrm{m}^{-1}$ & \multicolumn{2}{|c|}{1.600 ind. $\mathrm{m}^{-1}$} \\
\hline Individuos vivos por cuerda & 333 & 583,5 & \multirow{2}{*}{\multicolumn{2}{|c|}{$\begin{array}{c}1.225 \\
76,56 \% *\end{array}$}} \\
\hline Supervivencia porcentual & $83,25 \%{ }^{a, *}$ & $83,36 \%{ }^{a, *}$ & & \\
\hline 6 meses & 400 ind. $\mathrm{m}^{-1}$ & 700 ind. $\mathrm{m}^{-1}$ & 400 ind. $\mathrm{m}^{-1}$ & 700 ind. $\mathrm{m}^{-1}$ \\
\hline Individuos vivos por cuerda & 338 & 520 & 289 & 653 \\
\hline Supervivencia porcentual & $84,5 \%{ }^{b}$ & $74,29 \%^{b}$ & $72,25 \%(\#)$ & $93,29 \%(\#)$ \\
\hline 9 meses & 400 ind. $\mathrm{m}^{-1}$ & 700 ind. $\mathrm{m}^{-1}$ & 400 ind. $\mathrm{m}^{-1}$ & 700 ind. $\mathrm{m}^{-1}$ \\
\hline Individuos vivos por cuerda & 308,5 & 224 & 224 & 606 \\
\hline Supervivencia porcentual & $77,13 \%$ & $32 \%$ & $56 \%(\#)$ & $86,57 \%(\#)$ \\
\hline $\begin{array}{l}\text { Supervivencia porcentual total } \\
\text { (fase de pre-engorde y fase de engorde) }\end{array}$ & $77,13 \% *$ & $32 \%$ & $43 \%$ & $66,2 \% *$ \\
\hline
\end{tabular}


Los datos de supervivencia total resultantes de la aplicación de la ecuación (1) muestran que en la ESD los porcentajes se mantuvieron en todo el experimento, ya que en esta estrategia no se realizó desdoble (Tabla 2). Por el contrario, en la ECD los porcentajes de supervivencia fueron inferiores a los obtenidos cuando solo se consideró la etapa de engorde. En este análisis, los mayores porcentajes de supervivencia se observaron en la ESD$400(77 \%)$ y ECD-700 (66\%), siendo estos resultados independientes de la estrategia seleccionada (prueba de independencia $\chi 2, \mathrm{p}>0,05, \mathrm{GL}=1$ ).

\section{Análisis de crecimiento}

El análisis a los tres meses (primer muestreo) mostró que casi todas las series experimentales (tanto en talla como en peso) difieren significativamente entre sí con una relación inversa entre las medias y/o distribuciones y las densidades iniciales (Tabla 3). Las excepciones se presentaron en la tallas correspondientes a ESD-400 y ESD-700 (Tukey post-hoc, $\mathrm{p}>0,05$ ), y en los pesos secos correspondientes a ESD-700 y ECD-1.600 (Kruskal-Wallis-Dunn a posteriori, $\mathrm{p}>0,05, \mathrm{GL}=2$ ).

Dentro de la ESD no se observaron diferencias significativas en la talla a los tres (Welch, p > $0,05, \mathrm{GL}=193,58$ ) ni a los seis meses (Welch, $\mathrm{p}>0,05, \mathrm{GL}=197,34)($ Figura 2). En el último muestreo, la talla promedio en la ESD-400 presentó un valor de $66,58 \mathrm{~mm}$, siendo este registro significativamente mayor al observado en la ESD-700 (Welch, $\mathrm{p}=0,001, \mathrm{GL}=66,8$ ). Además de presentar un tamaño promedio significativamente mayor, la ESD-400 registró también la menor dispersión de tallas al momento de la cosecha entre las cuatro series experimentales ( $\mathrm{s}=$ 5,31). Para el peso seco dentro de esta estrategia, se observaron diferencias significativas a los tres (Mann-Whitney, $\mathrm{p}=0,002$ ) y a los nueve meses (Mann-Whitney, $\mathrm{p}=0$,0006) en favor de la ESD400 , la cual alcanzó al final de la experiencia un registro de 1,63 g (Figura 2).

En la ECD se observaron diferencias significativas en las tallas solo en la última fecha de muestreo (Welch, $p=0,0017, \mathrm{GL}=135,37)$ (Figura 3). El mayor crecimiento final se obtuvo en la ECD400 , con $62,53 \mathrm{~mm}$. Para el caso del peso no se presentaron diferencias significativas en ningún momento después del desdoble (Mann-Whitney, $\mathrm{p}>0,05)$ (Figura 3).

En la comparación de ambas estrategias al momento de cosecha, el mayor crecimiento en talla se observó en las cuerdas de la ESD, específicamente en la densidad más baja (400 ind. $\left.\mathrm{m}^{-1}\right)$,

Tabla 3. Talla y pesos promedio con su error estándar. Para cada fecha de muestreo, diferencias no significativas dentro de cada estrategia se denota con la misma letra. Diferencias no significativas en la comparación entre estrategias se denotan con *.

Table 3. Average size and weight with their standard error. For each sampling date, non-significant differences within each strategy are denoted with the same letter. Non-significant differences in the comparison between strategies are denoted with *

\begin{tabular}{|c|c|c|c|c|c|}
\hline & & \multicolumn{2}{|c|}{ Experiencia sin desdoble } & \multicolumn{2}{|c|}{ Experiencia con desdoble } \\
\hline & & 400 ind. $\mathrm{m}^{-1}$ & 700 ind. $\mathrm{m}^{-1}$ & 400 ind. $\mathrm{m}^{-1}$ & 700 ind. $\mathrm{m}^{-1}$ \\
\hline \multirow[t]{2}{*}{3 meses } & Talla (mm) & $41,06(4,32)^{\mathrm{a}}$ & $39,98(3,71)^{\mathrm{a}}$ & \multicolumn{2}{|c|}{$37,78(4,68)$} \\
\hline & Peso (g) & $0,2332(0,059)$ & $0,208(0,049)^{*}$ & \multicolumn{2}{|c|}{$0,197(0,09)^{*}$} \\
\hline \multirow[t]{2}{*}{6 meses } & Talla (mm) & $50,82(6,68)^{b}$ & $50,36(6,94)^{b}$ & $44,16(5,53)^{\mathrm{d}}$ & $43,93(6,5)^{d}$ \\
\hline & Peso (g) & $0,8727(0,25)^{\mathrm{c}}$ & $0,8124(0,26)^{\mathrm{c}}$ & $0,6338(0,19)^{\mathrm{e}}$ & $0,5817(0,23)^{\mathrm{e}}$ \\
\hline \multirow[t]{2}{*}{9 meses } & Talla (mm) & $66,83(5,31)$ & $62,39(8,03)^{*}$ & $62,53(5.97)^{*}$ & $58,62(8.88)$ \\
\hline & Peso $(\mathrm{g})$ & $1,63(0,47)$ & $1,34(0,465)^{*}$ & $1,17(0.303)^{f, *}$ & $1,15(0,5)^{\mathrm{f}, *}$ \\
\hline
\end{tabular}



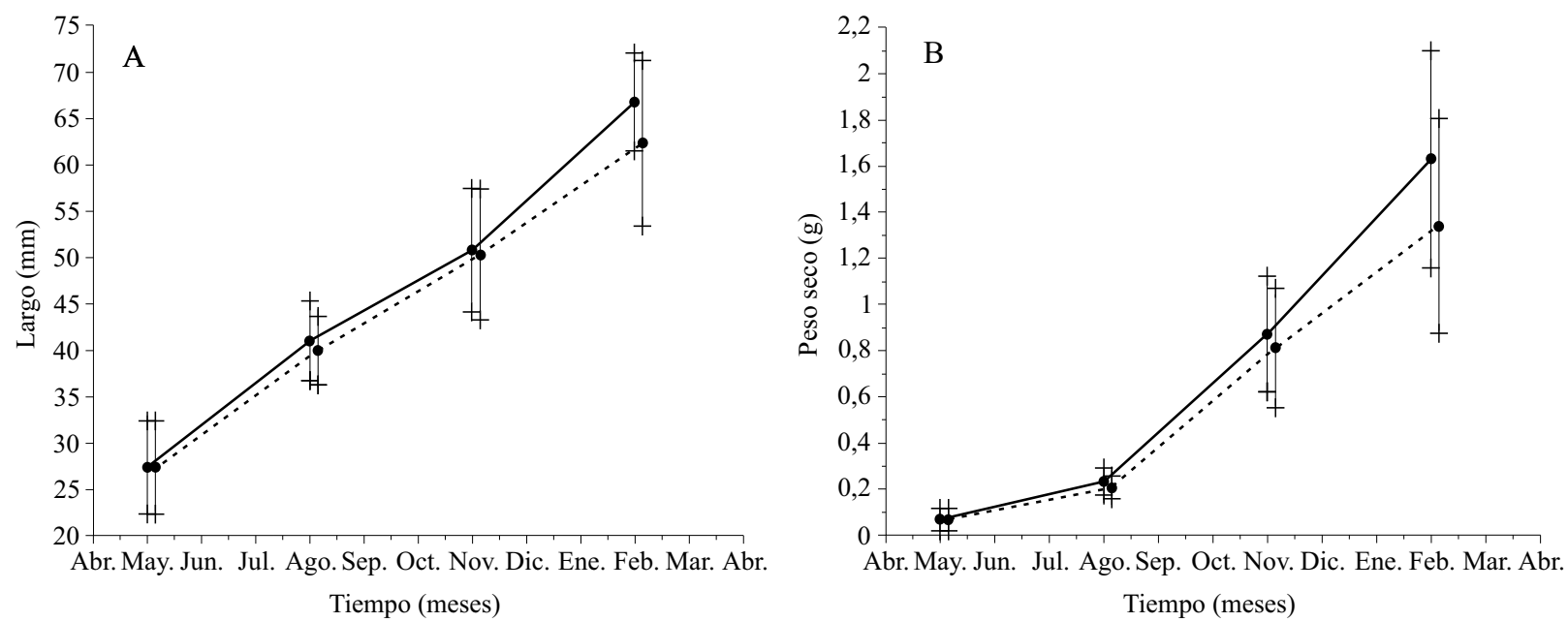

Figura 2. Evolución de crecimiento en ESD en talla (A) y peso seco (B) promedios. Línea continua: ESD-400. Línea punteada: ESD-700 (barras verticales: error estándar).

Figure 2. Evolution of growth in ESD in average size (A) and dry weight (B). Solid line: ESD-400. Dotted line: ESD-700 (vertical bars: standard error).

siendo este resultado significativamente mayor que las otras medias (Kruskal-Wallis, Dunn a posteriori, $\mathrm{p}<0,05, \mathrm{GL}=3$ ). El peso seco mostró la misma tendencia, con ESD-400 presentando el mayor peso final y diferencias significativas con las restantes observaciones (Kruskal-Wallis-Dunn a posteriori, $\mathrm{p}<0,05, \mathrm{GL}=3$ ).

\section{DISCUSIÓN Y CONCLUSIONES}

Los efectos de la densidad sobre los parámetro productivos generales han sido ampliamente estudiados en lugares donde el cultivo de mejillón se desarrolla de manera intensiva (Fuentes et al. 1992; Grant 1996; Fréchette y Bacher 1998; Alunno-Bruscia et al. 2000; Zaixso y Lizarralde 2001; Lauzon-Guay et al. 2005a, 2005b; Fréchette et al. 2010, 2011, 2013; Lachance-Bernard et al. 2010; Cubillo et al. 2012a; Bordon et al. 2014, entre otros).

En la Argentina, el desarrollo del cultivo intensivo del mejillón es aún una actividad pendiente. Los emprendimientos en la región costera del
Golfo San Jorge son esporádicos, a baja escala y a densidades iniciales no reguladas sistemáticamente. En este contexto, el objetivo de este trabajo consistió en estudiar el engorde y la supervivencia en cultivos experimentales con y sin la práctica de desdoble, a dos densidades iniciales de engorde.

A lo largo de la experiencia se registraron dos picos de Chl $a$, a mediados otoño (mayo) y en primavera (octubre y noviembre). El valor máximo registrado de MOP se observó en el mes de noviembre. Las concentraciones de Chl $a$ sufrieron marcadas disminuciones en los meses de invierno. Los valores máximos, tanto de Chl $a$ como de MOP, se registraron en el mes de noviembre y estuvieron en concordancia con un marcado aumento de temperatura durante ese mes. Estos resultados fueron consistentes con otros estudios realizados en el área (Akselman 1996; Fernández et al. 2005, 2008; Carreto et al. 2007).

En términos generales, durante los primeros tres meses de cultivo (temporada de invierno en la región) todos los animales multiplicaron su peso promedio por tres, y su talla promedio por 1,5. Este desarrollo individual puede deberse al 

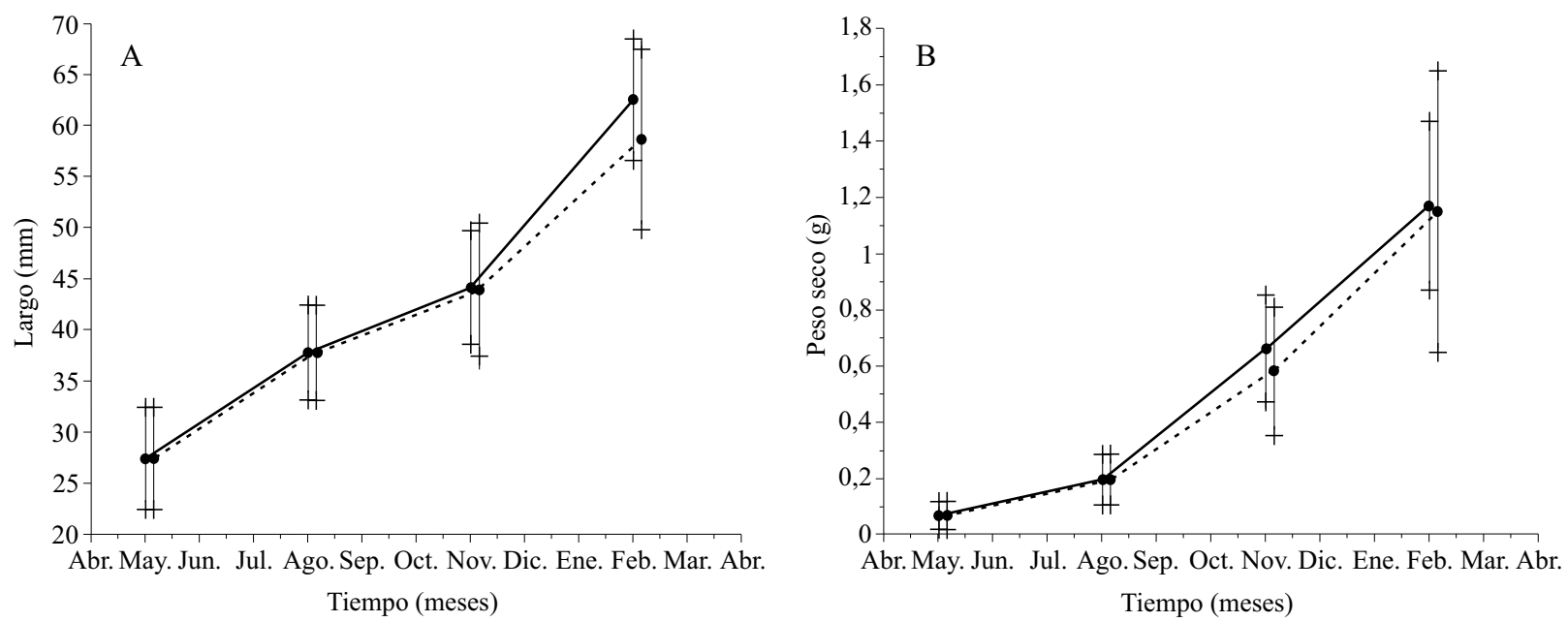

Figura 3. Evolución de crecimiento en ECD en talla (A) y peso seco (B) promedios. Línea continua: ECD-400. Línea punteada: ECD-700 (barras verticales: error estándar).

Figure 3. Evolution of ECD growth in average size (A) and dry weight (B). Solid line: ECD-400. Dotted line: ECD-700 (vertical bars: standard error).

estadío de vida de los animales dado que, al momento de siembra, los individuos eran juveniles (no más de 4 meses), una etapa en la que el crecimiento es muy marcado en cortos períodos de tiempo (Bayne 1976; Gosling 1992).

A los 6 meses (segundo muestreo), se registró un aumento en la temperatura y la cantidad de alimento disponible en la columna de agua (Chl $a$ y MOP), asociado con la temporada de primavera y el comienzo del verano. Esta mejora en las condiciones está en concordancia con el desarrollo gonadal y la primera madurez sexual de los individuos informados en el área de estudio en esta época del año (Isola 2017), lo que explica el aumento de peso promedio observado de todos los animales. En el último trimestre del experimento, las condiciones ambientales favorables observadas en los meses de primavera-verano explicarían el aumento de talla y peso registrado.

El análisis de las diferentes estrategias de cultivo planteadas en la experiencia mostró que la estrategia sin desdoble a bajas densidades (ESD400) brindó los mejores resultados en casi todas los parámetros de crecimiento estudiados. La supervivencia final presentó los valores más altos
$(77 \%)$, y tanto la talla (incremento de $39 \mathrm{~mm}$ ) como el pesos seco (1,6 g en promedio) mostraron valores significativamente mayores a los obtenidos en las series experimentales restantes.

En un experimento de engorde similar (sin desdoble) realizado en España durante en el cultivo del mejillón M. galloprovincialis durante 12 meses, los animales alcanzaron tallas similares y valores de peso seco inferiores a los valores observados en este trabajo para ESD-400 (PérezCamacho et al. 2013). Cabe señalar que en el trabajo citado los mejillones se cultivaron a densidades más altas y por un período mayor que en este trabajo (9 meses).

Los animales, independientemente de la técnica utilizada, alcanzaron tallas promedios superiores a $58 \mathrm{~mm}$ al final de la experiencia. Sin embargo, la ESD-400 presentó la mayor talla promedio $(66,58$ $\mathrm{mm}$ ) y el menor valor de dispersión (menor error estándar, $\mathrm{s}=5,31$ ). Estos valores sugieren un mejor crecimiento y tallas más uniformes al momento de la cosecha en esta serie experimental, a diferencia de lo observado en las restantes.

Diversas experiencias realizadas en la región patagónica muestran que el tiempo de cultivo 
requerido para alcanzar tallas superiores a $65 \mathrm{~mm}$ va de los 7 (Elvira et al. 2000) a los 9 meses (Bertolotti et al. 1987). El tiempo de cultivo observado en este trabajo ( 9 meses) es comparable a estos registros, y menor a lo generalmente empleado en otras partes del mundo (Uriarte 2008; Cubillo et al. 2012a; Díaz et al. 2019). Estos resultados muestran el potencial para el desarrollo del cultivo de mejillón en la región de estudio.

La densidad inicial óptima determinada en este trabajo es menor que la utilizada en países como Chile (Díaz et al. 2011) en el cultivo de mejillones del Género Mytilus, aunque similar a la utilizada en Brasil en el cultivo de mejillones del Género Perna (Bordon et al. 2014), y en el cultivo del mejillón $M$. platensis, en el Golfo San Matías, Chubut, Argentina (Pascual y Zampatti 1998; Elvira et al. 2000). Como consecuencia de la siembra a bajas densidades se reduce la competencia intraespecífica por alimento y espacio (Fréchette y Bacher 1998; Cubillo et al. 2012a), posibilitando un mayor desarrollo individual.

Los resultados de este trabajo muestran que la siembra de individuos de un tamaño de alrededor de $25 \mathrm{~mm}$ a una densidad inicial de 400 ind. $\mathrm{m}^{-1}$ a mediados de otoño (mayo en la región de estudio) permite obtener tamaño comercial al cabo de 9 meses. La densidad inicial, el crecimiento en talla y los tiempos requeridos para alcanzar estas tallas están dentro de los rangos observados en otras experiencias en la región patagónica (Bertolotti et al. 1987; Elvira et al. 2000). Sumado a esto, resultados de otras experiencias en la región mostraron que el cultivo por un período mayor a 12 meses genera pérdidas masivas debido al aumento de peso individual (Zaixso y Lizarralde 2001), estableciendo un período de cosecha que va de 9 a 12 meses desde la siembra de los animales.

Dado que en el área de estudio no existen emprendimientos continuos ni de gran escala, los resultados obtenidos en esta investigación constituyen una línea de base en relación a la evaluación de técnicas de manejo que permitan optimi- zar el crecimiento y la supervivencia. Se necesitarán futuros estudios, a una escala comercial, que contemplen parámetros productivos (rendimientos por metro de cuerda, índices de condición, diversos orígenes de la semilla, etc.) y aspectos ecosistémicos para determinar cómo el aumento en la escala de producción puede afectar la capacidad de carga del sistema (Ross et al. 2013). Sobre la base de los resultados del presente estudio, la estrategia de engorde sin desdoble a una densidad inicial de 400 ind. $\mathrm{m}^{-1}$ sería la recomendada para el cultivo de mejillón en la región, alcanzando una talla mayor a $66 \mathrm{~mm}$ al cabo de nueve meses de engorde y tallas más homogéneas al momento de la cosecha (menor error estándar observado). Por otra parte, el cultivo sin desdoble reduce las horas/hombre de trabajo (dado que no se practica desdoble). La información obtenida establece la estrategia de manejo adecuada y las densidades iniciales óptimas para maximizar el crecimiento y la supervivencia, generando conocimiento de base fundamental para futuros emprendimientos a mayor escala, contemplando el desarrollo sustentable de la actividad.

\section{AGRADECIMIENTOS}

Agradecemos a los docentes a cargo del criadero (hatchery) perteneciente a la Escuela Provincial $\mathrm{N}^{\circ} 16$, ubicada en la ciudad de Bahía Camarones, Chubut, por suministrar los juveniles necesarios para esta experiencia y a la Agencia Comodoro Conocimiento, Chubut, Argentina, por la provisión de la línea de cultivo para la realización del experimento.

Los autores agradecen los comentarios y sugerencias realizados por los revisores, los cuáles ayudaron a mejorar el trabajo y su presentación.

El trabajo de B. Marques fue apoyado por una beca doctoral tipo I financiada por el Consejo Nacional de Investigaciones Científicas y Técnicas (CONICET), Argentina. 


\section{REFERENCIAS}

Akselman, R. 1996. Estudios ecológicos en el Golfo San Jorge y adyacencias (Atlántico Sudoccidental): Distribución, abundancia y variación estacional del fitoplancton en relación a factores Físico-químicos y la dinámica hidrológica [tesis doctoral]. Buenos Aires: Facultad de Ciencias Exactas y Naturales, Universidad de Buenos Aires. 234 p.

Alunno-Bruscia M, Petraitis PS, Bourget E, FrÉCHETTE M. 2000. Body size-density relationship for Mytilus edulis in an experimental food-regulated situation. Oikos. 90 (1): 28-42.

BAYNE B. 1976. Marine mussels: their ecology and physiology. International Biological Programme. Vol. 10. Cambridge University Press. $411 \mathrm{p}$.

Bertolotti M, Lasta M, Zampatti E. 1987. Cultivo experimental de mejillón (Mytilus edulis platensis): características biológicas, técnicas y análisis económico de la actividad. Industria Cárnica Latinoamericana. 65: 42-54.

Bordon ICAC, Marques HLA, Alves JL, Rossi A, PECora IL. 2014. Influence of densities on the growth of brown mussel Perna perna L. cultivated in suspended socks at Caraguatatuba, southeastern Brazil. Aquac Int. 22 (2): 541-549.

Carreto Ji, Carignan MO, Montoya NG, Colleoni Cucchi AD. 2007. Ecología del fitoplancton en los sistemas frontales del Mar Argentino. En: CARRETo JI, BREMEC C, editores. El Mar Argentino y sus recursos pesqueros. Tomo 5. El ecosistema marino. Mar del Plata: Instituto Nacional de Investigación y Desarrollo Pesquero (INIDEP). p. 11-31.

Cubillo AM, Fuentes-Santos I, Peteiro LG, FERNÁNDEZ-REIRIZ MJ, LABARTA U. 2012c. Evaluation of self-thinning models and estimation methods in multilayered sessile animal populations. Ecosphere. 3 (8): 1-23.
Cubillo Am, Peteiro LG, Fernández-Reiriz MJ, LABARTA U. 2012a. Influence of stocking density on growth of mussels (Mytilus galloprovincialis) in suspended culture. Aquaculture. 342: 103-111.

Cubillo AM, Peteiro LG, Fernández-Reiriz MJ, LABarta U. 2012b. Density-dependent effects on morphological plasticity of Mytilus gallloprovincialis in suspended culture. Aquaculture. 338: 246-252.

Díaz C, Figueroa Y, Sobenes C. 2011. Effect of different longline farming designs over the growth of Mytilus chilensis (Hupé, 1854) at Llico Bay, VIII Región of Bio-Bio, Chile. Aquac Eng. 45 (3): 137-145.

Díaz C, Sobenes C, Machino S. 2019. Comparative growth of Mytilus chilensis (Hupé 1854) and Mytilus galloprovincialis (Lamarck 1819) in aquaculture longline system in Chile. Aquaculture. 507: 21-27.

Elvira M, Pascual M, Fernandez Cartes V, Zampatti E, Agulleiro I, Soria G. 2000. Crecimiento a talla comercial del mejillón, Mytilus edulis platensis, en cuerdas de cultivo en el paraje Bajo Oliveira (Golfo San Matías, Argentina). En: Proceedings de las IV Jornadas Nacionales de Ciencias del Mar, 11 al 15 de septiembre de 2000, Puerto Madryn, Argentina. p. 59.

Fernández M, Carreto Ji, Mora J, Roux A. 2005. Physico-chemical characterization of the benthic environment of the golfo San Jorge, Argentina. J Mar Biol Assoc UK. 85: 1317-1328.

Fernández M, Mora J, Roux A, Cucchi Colleoni DH, Gasparonia JC. 2008. New contribution on spatial and seasonal variability of environmental conditions of the golfo San Jorge benthic system, Argentina. J Mar Biol Assoc UK. 88 (2): 227-236.

FrÉCHETTE M, BACHER C. 1998. A modelling study of optimal stocking density of mussel populations kept in experimental tanks. J Exp Mar Biol Ecol. 219 (1): 241-255. 
Fréchette M, Bergeron P, Gagnon P. 1996. On the use of self-thinning relationships in stocking experiments. Aquaculture. 145: 91-112.

Fréchette M, Lachance-Bernard M, Daigle G. 2010. Body size, population density and factors regulating suspension-cultured blue mussel (Mytilus spp.) populations. Aquat Living Resour. 23 (3): 247-254.

FrÉChetTe M, LefaIVRe D. 1990. Discriminating between food and space limitation in benthic suspension feeders using self-thinning relationship. Mar Ecol Prog Ser. 65: 15-23.

Fréchette M, Urquiza JM, Daigle G, Maheux D, DumaIs JF. 2013. Self-thinning dynamics in experimental scallop populations. Aquac Int. 21 (3): 539-551.

FrÉCHETte M, Wilson JR, Bilodeau F, LACHANCE-BERnARD M. 2011. Production dynamics, self-thinning and profitability of blue mussel populations reared in suspension culture in Cascapédia Bay. En: Proceedings of Aquaculture Canada OM 2010 and Cold Harvest TM 2010. Mayo 16-19, St. John's, Newfoundland and Labrador. p. 30-33.

Fuentes J, Reyero I, Zapata C, Alvarez G. 1992. Influence of stock and culture site on growth rate and mortality of mussels (Mytilus galloprovincialis Lmk.) in Galicia, Spain. Aquaculture. 105 (2): 131-142.

Fuentes-Santos I, Cubillo AM, FernándeZReIriz JM, LABARTA U. 2014. Dynamic selfthinning model for sessile animal populations with multi-layered distribution. Rev Aquacult. 6 (2): 115-127.

GonzÁlez MM, Hernández V, SAnta Cruz G. 1974. Algunos aspectos de la tecnología de los cultivos marinos en Chile. Actas del Simposio sobre Acuicultura en América Latina Volumen 1 - Documentos de Investigación. 26 de noviembre al 2 de diciembre de 1974, Montevideo, Uruguay. FAO, Informes de Pesca.

GosLING E, editor. 1992. The mussel Mytilus: ecology, physiology, genetics and culture. Developments in Aquaculture and Fisheries
Science, 25. Amsterdam: Elsevier. 589 p.

GRANT J. 1996. The relationship of bioenergetics and the environment to the field growth of cultured bivalves. J Exp Mar Biol Ecol. 200 (12): 239-256.

GuiÑEZ R. 2005. Layering, the effective density of mussels and mass-density boundary curves. Oikos. 110 (1): 186-190.

INFOLEG. c2017. Infoleg.gob.ar, NJ: Infoleg; [actualizado 4 septiembre 2017; consultado 22 octubre 2019]. http://servicios.infoleg.gob.ar/ infolegInternet/anexos/275000-279999/2788 95/norma.htm.

Isola T. 2017. Aspectos tecnológicos y bio-ecológicos del asentamiento de mejillón, Mytilus edulis platensis, sobre colectores artificiales [tesis doctoral]. Bahía Blanca: Departamento de Biología, Bioquímica y Farmacia, Universidad Nacional del Sur. $153 \mathrm{p}$.

Isola T, ZAIXSO H. 2012. Efecto de la densidad de mejillón sobre la biomasa cosechable en cuerdas de engorde de tipo mixto (francés-español). En: Proceedings de las VIII Jornadas Nacionales de Ciencias del Mar. 3 al 7 de diciembre de 2012, Comodoro Rivadavia. p. 140.

Lachance-Bernard M, Daigle G, Himmelman JH, FrÉCHETTE M. 2010. Biomass-density relationships and self-thinning of blue mussels (Mytilus spp.) reared on self-regulated longlines. Aquaculture. 308 (1-2): 34-43.

Lauzon-Guay JS, Dionne M, Barbeau MA, HAMILTON DJ. 2005a. Effects of seed size and density on growth, tissue-to-shell ratio and survival of cultivated mussels (Mytilus edulis) in Prince Edward Island, Canada. Aquaculture. 250 (3-4): 652-665.

Lauzon-Guay J, Hamilton DJ, Barbeau MA. 2005b. Effect of mussel density and size on the morphology of blue mussels (Mytilus edulis) grown in suspended culture in Prince Edward Island, Canada. Aquaculture. 249 (14): 265-274.

Mann HB, Whitney DR. 1947. On a test of whether one of two random variables is sto- 
chastically larger than the other. Ann Math Stat. 18: 50-60.

PAnNé Huidobro S. 2015. Producción por Acuicultura en Argentina en el 2015. Dirección de Acuicultura. Dirección Nacional de Planificación Pesquera. Subsecretaría de Pesca y Acuicultura. Ministerio de Agroindustria. 9 p.

Pascual M, Zampatti E. 1998. Cultivos de moluscos bivalvos. En: BosCHI E, editor. El Mar Argentino y sus recursos. Tomo 2. Los moluscos de interés pesquero. Cultivos y estrategias reproductivas de bivalvos y equinoideos. Mar del Plata: Instituto Nacional de Investigación y Desarrollo Pesquero (INIDEP). p. 167-195.

PÉREZ-CAMACho A, LABARTA U, Vinseiro V, FERNÁNDEZ-REIRIZ MJ. 2013. Mussel production management: raft culture without thinning-out. Aquaculture. 406: 172-179.

PROCHILE. c2014. Oficina Comercial de Chile en Buenos Aires y Mendoza. Ministerio de Relaciones Exteriores; [consultado 22 octubre 2019]. https://www.prochile.gob.cl/wp-con tent/files_mf/1424888380PMP_Argentina_ Pesqueros_2014.pdf.

[RMCP] RED DE ForTALECIMIENTO PARA LA MARICultura Costera Patagónica. 2013. Relevamiento de la Actividad de maricultura en la Patagonia argentina. Documento Técnico. Vol. 1. $222 \mathrm{p}$.

Ross LG, Telfer TC, Falconer L, Soto D. 2013. Site selection and carrying capacities for inland and coastal aquaculture. FAO/Insti- tute of Aquaculture, University of Stirling, Expert Workshop -8 December 2010 Stirling, the United Kingdom of Great Britain and Northern Ireland. 295 p.

SOKAL RR, ROHLF FJ. 1995. Biometry: the principles of statistics in biological research. San Francisco: Freeman \& Co. 776 p.

STRICKLAND JDH, PARSON S. 1965. A manual of sea water analysis. Bull Fish Res Board Can. 125. $203 \mathrm{p}$.

URIARTE I. 2008. Estado actual del cultivo de moluscos bivalvos en Chile. Estado actual del cultivo y manejo de moluscos bivalvos y su proyección futura: factores que afectan su sustentabilidad en América Latina. Taller Técnico Regional de la FAO. p. 20-24.

ZaIXso HE, BALA JL. 1993. Crecimiento de mejillones cultivado en encordados franceses y españoles. Naturalia Patagónica Cienc Biol. 1 (1): 8-21.

Zaixso HE, LiZARralde Z. 1999. Efecto del método de encordado sobre el crecimiento y la pérdida de mejillones (Mytilus platensis d'Orb.) en cultivos suspendidos. Biol Pesq. 27: 47-62.

Zaixso HE, LiZARralde Z. 2001. Efectos del desdoble sobre la biomasa cosechable de Mytilus platensis D'Orb en cultivo. Rev Invest Desarr Pesq. 14: 109-123.

Recibido: 16-12-2019

Aceptado: 14-2-2020 
